Research Article

\title{
Convergence Analysis of a Fourier-Based Solution Method of the Laplace Equation for a Model of Magnetic Recording
}

\author{
John L. Fleming \\ Department of Mathematics and Computer Science, Duquesne University, 600 Forbes Avenue, \\ Pittsburgh, PA 15282, USA
}

Correspondence should be addressed to John L. Fleming, flemingj@duq.edu

Received 1 April 2007; Accepted 25 November 2007

Recommended by F. E. Udwadia

When engineers model the magnetostatic fields applied to recording heads of computer hard drives due to a magnetic recording medium, the solution of Laplace's equation must be found. A popular solution method is based on Fourier analysis. However, due to the geometry of the read head model, an interesting mathematical problem arises. The coefficients for the Fourier series solution of the desired magnetic potential satisfy an infinite system of linear equations. In practice, the infinite system is truncated to a finite system with little consideration for the effect this truncation has on the solution. The paper will provide a proper understanding of the underlying problem and a formal analysis of the effect of truncation.

Copyright $@ 2008$ John L. Fleming. This is an open access article distributed under the Creative Commons Attribution License, which permits unrestricted use, distribution, and reproduction in any medium, provided the original work is properly cited.

\section{Introduction}

Engineering models of magnetic recording seek to simulate the magnetic potential that a shielded magnetoresistive head will experience as it passes over a magnetized recording medium. Finding the magnetic potential requires the solution of the Laplace equation. Often, a simple rectangular-type geometry is employed which naturally leads to the use of Fourierbased solutions. The desired potential can be found formally by some fairly straightforward calculations. However, in spite of the apparent simplicity, there is an important mathematical issue which arises in these calculations. Since the models involve a transition from an empty half-space to slot with a finite width, the Fourier coefficients of the solution satisfy an infinite system of linear equations. In order to compute a solution, the infinite system must be truncated to a finite system. From a mathematical and numerical analysis point of view, the effect of this truncation is not well understood or adequately explained. The main goal of this paper 


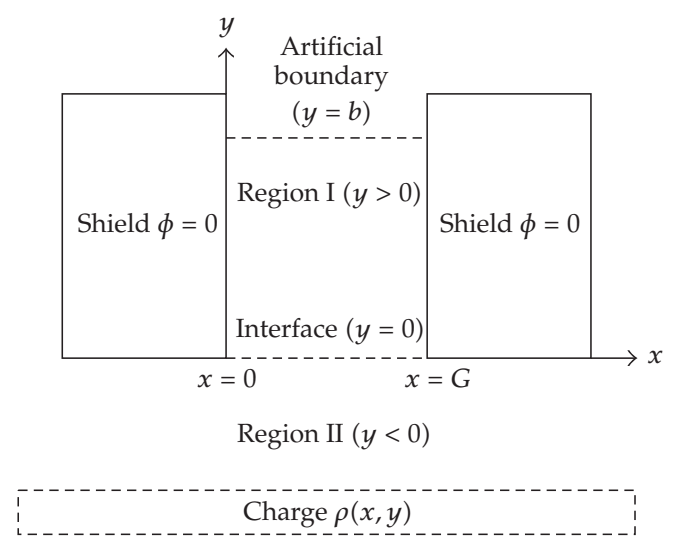

Figure 1: Diagram of solution $S$ domain for the variational problem.

is to provide the proper mathematical context under which the effect of the truncation can be characterized.

\subsection{Problem description}

Consider Laplace's equation $\Delta \phi=0$ in a two-dimensional setting. The solution $\phi$ represents a magnetic scalar potential. Further, assume that the $x y$-plane is divided into two half-spaces. In the lower half-space $(y<0)$, there is an empty space containing a charge which will induce a potential. In the upper half-space $(y>0)$, assume that there is a perfect magnetic conductor with a gap of empty space from $x=0$ to $x=G$. The empty space in region I will be referred to as "the gap," while the conducting material in region I will be called "the shields." Note that the shields are assumed to extend to infinity in both the $x$ - and $y$-directions. The geometry indicates that the potential is only nonzero in the lower half-space and in the gap between the shields in the upper half-space (see Figure 1). Also, note that the solution will satisfy a Dirichlet boundary condition $(\phi=0)$ at any interface with the shields.

A special boundary condition will be provided at the interface between region I and region II $(y=0)$. The boundary condition will ensure continuity of the potential and its normal derivative across the interface and also will properly reflect the influence of the charge in region II on the potential in region I. In addition, an artificial boundary will be placed in the gap at some positive value $y=b$ inside the gap in region I. The artificial boundary will create a finite rectangular domain which will be used later in the paper for the analysis of the variational form of the problem. The region within the gap, above the interface and below the artificial boundary, will be denoted as $S=[0, G] \times[0, b]$. The boundary of the region $S$ is denoted as $\Gamma$. In particular, the portion of the boundary at $y=b$ is denoted as $\Gamma_{1}$, while the portion of the boundary at $y=0$ is denoted as $\Gamma_{2}$.

The geometry described here has been used as a simplified model of the read process in magnetic recording [1-4]. A very similar situation using the Helmholtz equation is used to describe the scattering of plane waves from a groove in a perfect electric conductor $[5,6]$. Another similar situation occurs when dealing with waveguide junctions $[7,8]$. An approach of basic separation of variables allows us to find the form of the solutions in the lower and upper half-spaces in terms of their Fourier components. The aim is to find the solution of the Laplace 
equation in the gap (region I) of the upper half-space due to a charge which exists outside the gap in the lower half-space. However, to completely describe the solution in the gap of the upper half-space, it is necessary to study closely what occurs at the interface between the lower half-space and the gap $(y=0)$.

\subsection{Focus of the paper}

The paper will show how to produce the Fourier solution of the potential $\phi$ in the gap region described above. We will see that the solution method produces an infinite linear system of equations whose solution represents the Fourier coefficients of the potential in region I. The solution to the infinite system is approximated by solving a finite truncated version of the linear system. From a mathematical point of view, the truncation needs to be validated. In principle, there is no guarantee that the finite version even has a unique solution. Also, given the existence of the solution, it must be confirmed that the finite problem converges to the actual solution as the size of the finite system increases. Finally, if the problem does converge, we would like to characterize the rate at which the finite Fourier series converges to the actual series solution.

As we will see below, the nature of the infinite system is not easily conducive to mathematical or numerical analysis. As a novel approach, the paper will consider the variational solution of the problem which will provide an alternative avenue for analysis. It will be shown that in the proper context, the variational formulation is equivalent to the truncated finite version of the Fourier approach. Once this equivalence is established, the power of functional analysis will allow for a fairly straightforward analysis of the Fourier-based solution in the context of the variational solution.

The intent of the paper is not only to analyze the convergence of the finite approximations of the infinite linear system, but also to provide a perspective on the problem which is accessible to those familiar with functional analysis. The same theory which describes the convergence of finite element approximations will ultimately be applied to the finite Fourier-based approximations. Another benefit of this unique perspective is that it will allow for the description of the convergence properties without any need for direct analysis of the infinite linear system itself. One will simply need to determine how well the finite Fourier expansions are able to approximate the functions which are in the solution space of the variational problem.

\section{Fourier solution}

The Fourier-based solution method for the geometry in Figure 1 was offered in $[2,3]$. The method presented, or very similar methods, has been used within several models $[1,5,6]$. These papers offer a pragmatic method for finding the solution with Fourier theory, but they offer no mathematical justification of some important details of the method. In particular, as will be shown below, the solution of the Fourier methods satisfies an infinite linear system of equations. Approximate solutions are found by truncating these systems, but the mathematical analysis of this approach is lacking. Some analysis of a similar problem involving transitions in waveguides has been done where the infinite system is analyzed directly [8]. Some analysis of infinite matrices arising from solutions to Poisson's equation has been done[9, 10]. Also, analysis of infinite matrices using a matric operator technique for scattering problems has been done [11]. None of the mentioned works considers the alternative approach for the analysis using the variational form of the problem. 
The Fourier solution is found by studying what happens at the transition from region I to region II. In region I, the solution will have the following form:

$$
\phi_{\mathrm{I}}=\sum_{n=1}^{\infty} A_{n} \sin \left(\frac{n \pi x}{G}\right) \mathrm{e}^{-(n \pi / G) y} .
$$

Since the support of the potential in the $x$-direction is the interval from 0 to $G$, a Fourier sine series can be used. On the other hand, in region II the solution has the form

$$
\phi_{\mathrm{II}}=\int_{-\infty}^{+\infty}\left(B\left(k_{x}\right) e^{-\kappa y}+C\left(k_{x}\right) \mathrm{e}^{\kappa y}\right) \mathrm{e}^{2 \pi i k_{x} x} d k_{x}
$$

where $\mathcal{\kappa}=\left|2 \pi k_{x}\right|$. Since the support in the $x$-direction is $(-\infty, \infty)$ in region II, a Fourier transform must be used. The interesting part of the problem comes from coupling the two forms of the solution together at the gap interface.

Recall that the main problem is to compute the potential in region I given a charge distribution $\rho$ in region II. In terms of the Fourier approach, we must compute the Fourier coefficients $A_{n}$ in (2.1).

\subsection{Finite Fourier approximate solution method}

The finite Fourier method to compute the Fourier coefficients has been presented in multiple papers $[2,3]$. The solution comes from an examination of what happens at the interface between the gap in region I and the lower half-plane of region II. The two forms of the solution will satisfy some standard continuity conditions. tion:

First, the potential is continuous at the gap interface. This fact gives the following equa-

$$
\left.\phi_{\mathrm{I}}\right|_{y=0}=\left.\phi_{\mathrm{II}}\right|_{y=0} .
$$

Second, the normal derivative $(\partial / \partial y)$ is continuous across the gap interface. Hence, we have

$$
\left.\frac{\partial \phi_{\mathrm{I}}}{\partial y}\right|_{y=0}=\left.\frac{\partial \phi_{\mathrm{II}}}{\partial y}\right|_{y=0}
$$

The $A_{n}$ 's are computed by applying the continuity conditions to the two forms of the solution (2.1) and (2.2).

The first continuity equation gives the following:

$$
\sum_{n=1}^{\infty} A_{n} \sin \left(\frac{n \pi x}{G}\right)=\int_{-\infty}^{+\infty}(B+C) \mathrm{e}^{2 \pi i k_{x} x} d k_{x}
$$

or

$$
\sum_{n=1}^{\infty} A_{n} \sin \left(\frac{n \pi x}{G}\right)=\mathcal{F}_{x}^{-1}(B+C)
$$


Taking the Fourier transform in the $x$-direction of both sides gives

$$
\sum_{n=1}^{\infty} A_{n} \mathcal{F}_{x}\left(\sin \left(\frac{n \pi x}{G}\right)\right)=(B+C)
$$

or

$$
\mathcal{F}_{x}\left(\phi_{\mathrm{I}}\right)=B+C \text {. }
$$

Note that both the $A_{n}{ }^{\prime}$ s and $C$ are unknown. The $B$ can be computed directly using a Green function and the known charge distribution $\rho$ in region II. Hence, it is necessary to eliminate $C$ which allows for the determination of the $A_{n}{ }^{\prime} \mathrm{s}$ in terms of the known quantity $B$. The second continuity condition allows for the elimination of $C$ from the problem.

The second continuity condition gives the following:

$$
\sum_{n=1}^{\infty}\left(\frac{-n \pi}{G}\right) A_{n} \sin \left(\frac{n \pi x}{G}\right)=\int_{-\infty}^{+\infty}(-\kappa B+\kappa C) \mathrm{e}^{2 \pi i k_{x} x} d k_{x}
$$

or

$$
\sum_{n=1}^{\infty}\left(\frac{-n \pi}{G}\right) A_{n} \sin \left(\frac{n \pi x}{G}\right)=\mathcal{F}_{x}^{-1}(-\kappa B+\kappa C) .
$$

Multiplying both sides of the previous equation by $\sin (m \pi x / G)$ and integrating them from 0 to $G$ give the following:

$$
-\left(\frac{2}{G}\right)\left(\frac{m \pi}{G}\right) A_{m}=\int_{0}^{G} \mathcal{F}_{x}^{-1}(-\kappa B+\kappa C) \sin \left(\frac{m \pi x}{G}\right) d x .
$$

Solve for $C$ in (2.8) and substitute it into (2.11) to arrive at the following:

$$
\frac{-2 m \pi}{G^{2}} A_{m}=\int_{0}^{G} \mathcal{F}_{x}^{-1}\left[-2 \kappa B+\sum_{n=1}^{\infty} A_{n} \mathcal{F}_{x}\left(\sin \left(\frac{n \pi x}{G}\right)\right)\right] \sin \left(\frac{m \pi x}{G}\right) d x .
$$

By Parseval's formula, we have

$$
\frac{-2 m \pi}{G^{2}} A_{m}=-B_{m}+\sum_{n=1}^{\infty} K_{m n} A_{n}
$$

where

$$
\begin{gathered}
B_{m}=\int_{-\infty}^{+\infty} 2 B \mathcal{F}_{x}^{-1}\left(\sin \left(\frac{m \pi x}{G}\right)\right) d k_{x} \\
K_{n m}=\int_{-\infty}^{+\infty} \kappa F_{x}\left(\sin \left(\frac{n \pi x}{G}\right)\right) \mathcal{F}_{x}^{-1}\left(\sin \left(\frac{m \pi x}{G}\right)\right) d k_{x} .
\end{gathered}
$$

Thus, we have a formulation of the Fourier coefficients in terms of the Fourier transform $B$. However, we see that the Fourier coefficients of $\phi_{I}$ satisfy an infinite system of linear equations given by (2.13). Denote the infinite system as

$$
\tilde{K}\{\bar{A}\}=\{\bar{B}\},
$$

where $\tilde{K}_{n m}=K_{n m}+\left(-2 m \pi / G^{2}\right) \delta_{n m}$. 


\subsection{The issue of truncation}

For purposes of computation, such an infinite system does little good. An obvious response to deal with the situation is to solve a truncated version of the infinite matrix

$$
\widetilde{K_{N}}\left\{\overline{A_{N}}\right\}=\left\{\overline{B_{N}}\right\} .
$$

Even though this seems to be the logical approach to the infinite matrix issue from a computational perspective, the truncation leads to some important mathematical questions.

(1) Is the truncated matrix invertible?

(2) If the truncated matrix is invertible, does the finite version converge to the actual solution?

(3) If the finite version of the matrix converges to the actual solution, what is the convergence rate?

These very important questions must be answered to have some reasonable level of trust in the solution method. The problem is how to answer such questions about the truncation of the infinite matrix. Direct analysis of the infinite system is one approach, but an alternative provided below is to study the variational form of the problem and compare it to the Fourier method.

\section{Variational formulation}

The variational approach not only provides a basis for mathematical proofs of existence and uniqueness, but also provides a basis for robust numerical methods including the finite element method. First, the variational form of the problem will be derived. Second, the variational form will be shown to have a unique solution. Finally, it will be shown that if the variational form is applied to a particular space of functions, then the result is equivalent to the truncated Fourier method. Once this equivalence is established, the analysis of the truncated Fourier method can be performed by analyzing the equivalent variational form.

\subsection{Derivation of the variational form}

For the variational formulation, we will use the finite solution domain. Look for a solution of $\nabla u=0$ in the rectangular region $S=[0, G] \times[0, b]$. We will use $u$ to represent the solution of the variational problem. Ultimately, the solution will be equivalent to the potential $\phi_{\mathrm{I}}$ found by the Fourier approach. Recall that $\Gamma$ will denote the boundary of $S$. To enforce the boundary conditions $u(0, y)=u(G, y)=0$, we will look for a solution in the space of functions

$$
\widetilde{H}_{0}^{1}(S)=\left\{w \in H^{1}(S) \mid w(0, y)=w(G, y)=0\right\} .
$$

The weak form of the problem is constructed as follows. over $S$ :

(1) Multiply both sides of the equation $\nabla u$ by a function $w$ in $\widetilde{H}_{0}^{1}(S)$ and integrate them

$$
\int_{S} \Delta u w d S=0
$$


(2) Apply integration by parts:

$$
-\int_{S} \nabla u \cdot \nabla w d S+\int_{\Gamma} \frac{\partial u}{\partial n} w d \Gamma=0 .
$$

Here, we have the weak form or the variational form of the problem. We must examine the boundary integral term of the weak form of the equation. The boundary conditions must be constructed for the interface between region I and region II as well as for truncating region I domain to be finite.

We know that $w(0, y)=w(G, y)=0$, but we must also specify boundary conditions on $\Gamma_{1}(y=b)$ and $\Gamma_{2}(y=0)$. First, on $\Gamma_{1}$ we know that the solution will be of the form

$$
u=\sum_{n=1}^{\infty} A_{n} \sin \left(\frac{n \pi x}{G}\right) \mathrm{e}^{-(n \pi / G) y} .
$$

Therefore, on $\Gamma_{1}$ we have

$$
\frac{\partial u}{\partial n}=\frac{\partial u}{\partial y}=\sum_{n=1}^{\infty}\left(\frac{-n \pi}{G}\right) A_{n} \sin \left(\frac{n \pi x}{G}\right) \mathrm{e}^{-(n \pi / G) b} .
$$

Hence, a Dirichlet to Neumann map $T_{1}(u)$ can be defined on $\Gamma_{1}$ by

$$
\frac{\partial u}{\partial n}=T_{1}(u)=\sum_{n=1}^{\infty}\left(\frac{-n \pi}{G}\right) A_{n} \sin \left(\frac{n \pi x}{G}\right) \mathrm{e}^{-(n \pi / G) b .}
$$

On the boundary $\Gamma_{2}$, we must refer back to the second matching condition:

$$
\frac{\partial u}{\partial y}=\mathcal{F}_{x}^{-1}(-\kappa B+\kappa C)
$$

Again, solve (2.7) for $C$ and substitute it into the previous equation to get

$$
\frac{\partial u}{\partial y}=\mathcal{F}_{x}^{-1}\left(-2 \kappa B+\kappa \mathcal{F}_{x}(u)\right)
$$

or

$$
\frac{\partial u}{\partial y}=\mathcal{F}_{x}^{-1}\left(\kappa \mathcal{F}_{x}(u)\right)+g
$$

where $g=-2 \mathcal{F}_{x}^{-1}(\kappa B)$.

Hence, a Dirichlet to Neumann map $T_{2}(u)$ can be defined on $\Gamma_{2}$ as follows:

$$
\frac{\partial u}{\partial n}=T_{2}(u)+g=-\mathcal{F}_{x}^{-1}\left(\kappa \mathcal{F}_{x}(u)\right)-g .
$$

Now, put the maps $T_{1}(u)$ and $T_{2}(u)$ into the weak form of (3.3):

$$
-\int_{S} \nabla u \cdot \nabla w d S+\int_{\Gamma_{1}} T_{1}(u) w d \Gamma+\int_{\Gamma_{2}} T_{2}(u) w d \Gamma=\int_{\Gamma_{1}} g w d \Gamma .
$$

The weak form of the equation defines a bilinear form

$$
a(u, w)=-\int_{S} \nabla u \cdot \nabla w d S+\int_{\Gamma_{1}} T_{1}(u) w d \Gamma+\int_{\Gamma_{2}} T_{2}(u) w d \Gamma
$$


and a bounded linear functional

$$
\langle g, w\rangle=\int_{\Gamma_{1}} g w d \Gamma
$$

The solution of the variational form of the problem is a function $u \in \widetilde{H}_{0}^{1}(S)$ such that

$$
a(u, w)=\langle g, w\rangle
$$

for all $w \in \widetilde{H}_{0}^{1}(S)$.

\subsection{Existence and uniqueness}

The weak or variational formulation is very useful for proving existence and uniqueness of the problem at hand. The established theory for elliptic differential equations can be used here as in the following theorem.

Theorem 3.1. The variational problem (3.14) has a unique solution in $\widetilde{H}_{0}^{1}(S)$.

The existence/uniqueness proof can be found in [12]. The proof establishes the continuity and coercivity of the variational form in order to apply the Lax-Milgram theorem to verify existence and uniqueness.

The unique solution $u$ of the variational problem represents the potential $\phi_{\mathrm{I}}$ in region I. The finite Fourier approach produces an approximation of $\phi_{\mathrm{I}}$. At this point, the formal variational solution result can be used to analyze the approximate Fourier solution.

\section{The finite Fourier method versus the variational form}

\subsection{Existence of the finite Fourier solution}

The connection between the finite Fourier series method and the variational formulation can be found by considering subspaces of $\widetilde{H}_{0}^{1}(S)$. If a space $V_{N}$ is a subspace of $\widetilde{H}_{0}^{1}(S)$, then there is a unique solution to the variational problem over the subspace as well, because continuity and coercivity apply on the subspace in the same way as on the entire space [13]. Using this idea and Theorem 3.1, we want to compare the Fourier solution method and the variational form. The first conclusion which can be drawn from the comparison is that the truncated matrix from the Fourier method is invertible. We will see that this fact is just a simple corollary of the uniqueness result for the variational problem.

Define a finite subspace of $\widetilde{H}_{0}^{1}(S)$ :

$$
V_{N}=\left\{u_{N} \in \widetilde{H}_{0}^{1}(S) \mid u_{N}=\sum_{n=1}^{N} A_{n} \sin \left(\frac{n \pi x}{G}\right) \mathrm{e}^{(-n \pi / G) y}\right\}
$$

There is a unique solution $u_{N}$ to the variational problem in this space which satisfies

$$
a\left(u_{N}, v\right)=\langle g, v\rangle
$$


for all functions $v$ in the space $V_{N}$. If we examine the variational form of the problem when the functions come from the subspace $V_{N}$, the connection can be made to the finite Fourier solution.

Solve the variational form of the problem in $V_{N}$ using the Galerkin method. Let $v_{m}=$ $\sin (m \pi x / G) \mathrm{e}^{(-m \pi / G) y}$ and carry out the following calculations:

$$
\begin{aligned}
-\int_{S} \nabla u_{N} \cdot \nabla v_{m} d S & =\int_{0}^{b} \frac{2}{G}\left(\frac{m \pi}{G}\right) A_{m} \mathrm{e}^{(-m \pi / G) y} d y \\
& =\left[\left(\frac{m \pi}{G}\right)\left(\frac{2}{G}\right) A_{m} \mathrm{e}^{(-2 m \pi / G) b}-\left(\frac{m \pi}{G}\right)\left(\frac{2}{G}\right) A_{m}\right] \\
\int_{\Gamma_{1}} T_{1}\left(u_{N}\right) v_{m} d \Gamma & =\int_{0}^{G}\left[\sum_{n=1}^{N}-\left(\frac{n \pi}{G}\right) A_{n} \sin \left(\frac{n \pi x}{G}\right) \cdot \mathrm{e}^{(-n \pi / G) b}\right] \sin \left(\frac{m \pi x}{G}\right) \mathrm{e}^{(-m \pi / G) b} d x \\
& =-\left(\frac{m \pi}{G}\right)\left(\frac{2}{G}\right) A_{m} \mathrm{e}^{(-2 m \pi / G) b}, \\
\int_{\Gamma_{2}} T_{2}\left(u_{N}\right) v_{m} d \Gamma & =-\int_{0}^{G} \mathcal{F}_{x}^{-1}\left(\kappa \mathcal{F}_{x}\left(u_{N}\right)\right) \sin \left(\frac{m \pi x}{G}\right) d x \\
& =\int_{-\infty}^{\infty} \kappa \mathcal{F}_{x}\left(u_{N}\right) \mathcal{F}_{x}^{-1}\left(\sin \left(\frac{m \pi x}{G}\right)\right) d k_{x} .
\end{aligned}
$$

Continuing with the last integral,

$$
\begin{aligned}
\int_{-\infty}^{\infty} \mathcal{F}_{x}\left(u_{N}\right) \mathcal{F}_{x}^{-1}\left(\sin \left(\frac{m \pi x}{G}\right)\right) d k_{x} \\
=\sum_{n=1}^{N} \int_{-\infty}^{\infty} A_{n} \mathcal{F}_{x}\left(\sin \left(\frac{n \pi x}{G}\right)\right) \mathcal{F}_{x}^{-1}\left(\sin \left(\frac{m \pi x}{G}\right)\right) d k_{x} .
\end{aligned}
$$

Notice that this last expression is exactly

$$
-\sum_{n=1}^{N} K_{n m} A_{n}
$$

Putting all the integrations together, we get

$$
a\left(u_{N}, v_{m}\right)=-\left(\frac{m \pi}{G}\right)\left(\frac{2}{G}\right) A_{m}-\sum_{n=1}^{N} K_{n m} A_{n} .
$$

Also, note that

$$
\left\langle g, v_{m}\right\rangle=-\int_{-\infty}^{\infty} 2 B \mathcal{F}_{x}^{-1}\left(\sin \left(\frac{m \pi x}{G}\right)\right) d k_{x}
$$


which is exactly $-B_{m}$ from the Fourier method. Therefore, we see now that by solving the variational problem with the Galerkin method,

$$
a\left(u_{N}, v_{m}\right)=\left\langle g, v_{m}\right\rangle
$$

produces the exact same matrix as the truncated matrix from the Fourier method.

$$
\widetilde{K_{N}}\left\{\overline{A_{N}}\right\}=\left\{\overline{B_{N}}\right\} \text {. }
$$

Therefore, we have the desired equivalence between the finite Fourier method and the variational form of the problem on the subspace $V_{N}$. In particular, the solution $\overline{A_{N}}$ of (4.9) is the coefficients of $u_{N}$. Hence, we can answer the first question about the Fourier method. The truncated matrix is invertible. Since the variational form has a unique solution in $V_{N}$, we must conclude that the finite system representing the solution must be nonsingular.

\subsection{Convergence of Fourier method solution}

We can use analysis of the variational formulation on the subspace $V_{N}$ to determine the convergence properties of the Fourier series method.

Theorem 4.1. The finite Fourier method converges to the true solution of the problem in $\widetilde{H}_{0}^{1}(S)$.

Proof. Cea's lemma [13] states that the solution $u_{N}$ of the variational problem in $V_{N}$ is the best possible approximation of the true solution $u$ in $\widetilde{H}_{0}^{1}(S)$ :

$$
\left\|u-u_{N}\right\| \leq C\left(\inf _{\tilde{u} \in V_{N}}\|u-\tilde{u}\|\right) .
$$

By letting $N \rightarrow \infty$, we see that the finite Fourier method will converge to the actual solution.

Also, Cea's lemma allows us to answer the third question about the Fourier method regarding the convergence rate. Cea's lemma indicates that the solution $u_{N}$ from the subspace $V_{N}$ is the best possible approximation of the true solution $u$ in $\widetilde{H}_{0}^{1}(S)$. Therefore, we need to determine how "close" functions in $V_{N}$ can be to functions in $\widetilde{H}_{0}^{1}(S)$ with respect to their norm.

It can be shown that the approximations $u_{N}$ will converge to the actual series solution

$$
u=\sum_{n=1}^{\infty} A_{n} \sin \left(\frac{n \pi x}{G}\right) \mathrm{e}^{(-n \pi / G) y}
$$

with error of order $N^{-1}$ in $\widetilde{H}_{0}^{1}(S)$. The key to verifying this is to show that the trace of the first derivatives of $u$ is actually integrable on the boundary $\Gamma_{2}$. This can be done since the solution of the variational form of the problem can be shown to exist in the space $H^{3 / 2}(S)$ [14]. Therefore, the solution has enough regularity to conclude that $\left\|u-u_{n}\right\| \leq C / N$ [15]. 


\section{Conclusion}

At transitions of rectangular regions, Fourier-based solutions of differential equations will produce an infinite system of linear equations. In this paper, the Fourier-based computation of a Laplace equation inside a gap between two parallel perfect conductors is considered. In particular, the Fourier coefficients of the solution satisfy an infinite system of linear equations. Approximation techniques truncate this system without mathematical justification. The paper offered a method of analysis of the Fourier method using the variational form of the problem. We have seen that this approach proves that the truncation of the infinite linear system will always have a solution and the truncation will converge as the number of unknowns is increased.

\section{References}

[1] D. E. Heim, "On the track profile in magnetoresistive heads," IEEE Transactions on Magnetics, vol. 30, no. 4, pp. 1453-1464, 1994.

[2] H. A. Shute, D. T. Wilton, and D. J. Mapps, "Approximate Fourier coefficients for gapped magnetic recording heads," IEEE Transactions on Magnetics, vol. 35, no. 4, pp. 2180-2186, 1999.

[3] Y. Suzuki and C. Ishikawa, "A new method of calculating the medium field and the demagnetizing field for MR heads," IEEE Transactions on Magnetics, vol. 34, no. 4, pp. 1513-1515, 1998.

[4] S. X. Wang and A. M. Taratorin, Magnetic Information Storage Technology, Academic Press, San Diego, Calif, USA, 1999.

[5] H. J. Eom, T. J. Park, and K. Yoshitomi, "An analytic solution for the transverse-magnetic scattering from a rectangular channel in a conducting plane," Journal of Applied Physics, vol. 73, no. 7, 1993.

[6] M. A. Morgan and F. K. Schwering, "Mode expansion solution for scattering by a material filled rectangular groove," Progress in Electromagnetic Research, vol. 18, pp. 1-17, 1998.

[7] S. W. Lee, W. R. Jones, and J. J. Campbell, "Convergence of numerical solutions of iris-type discontinuity problems," IEEE Transactions on Microwave Theory and Techniques, vol. 19, no. 6, pp. 528-536, 1971.

[8] M. Leroy, "On the convergence of numerical results in modal analysis," IEEE Transactions on Antennas and Propagation, vol. 31, no. 4, pp. 655-659, 1983.

[9] T. N. Phillips and A. R. Davies, "On semi-infinite spectral elements for Poisson problems with reentrant boundary singularities," Journal of Computational and Applied Mathematics, vol. 21, no. 2, pp. 173-188, 1988.

[10] T. N. Phillips, "Fourier series solutions to Poisson's equation in rectangularly decomposable regions," IMA Journal of Numerical Analysis, vol. 9, no. 3, pp. 337-352, 1989.

[11] I. Petrusenko, "Mode diffraction: analytical justification of matrix models and convergence problems," in Proceedings of the 11th International Conference on Mathematical Methods in Electromagnetic Theory (MMET'06), pp. 332-337, Kharkov, Ukraine, June 2006.

[12] J. L. Fleming, "Existence and uniqueness of the solution of the laplace equation from modeling magnetic recording," Applied Mathematics E-Notes, vol. 8, pp. 17-24, 2008.

[13] B. D. Reddy, Introductory Functional Analysis, vol. 27 of Texts in Applied Mathematics, Springer, New York, NY, USA, 1998.

[14] S. A. Nazarov and B. A. Plamenevsky, Elliptic Problems in Domains with Piecewise Smooth Boundaries, vol. 13 of De Gruyter Expositions in Mathematics, Walter de Gruyter, Berlin, Germany, 1994.

[15] G. P. Tolstov, Fourier Series, Dover, New York, NY, USA, 1962. 


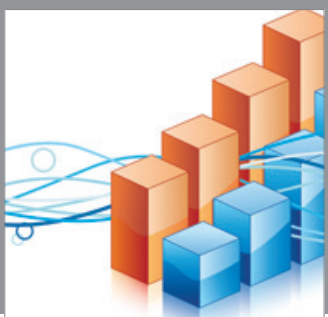

Advances in

Operations Research

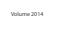

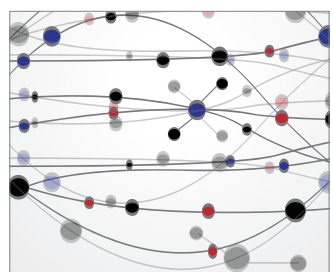

\section{The Scientific} World Journal
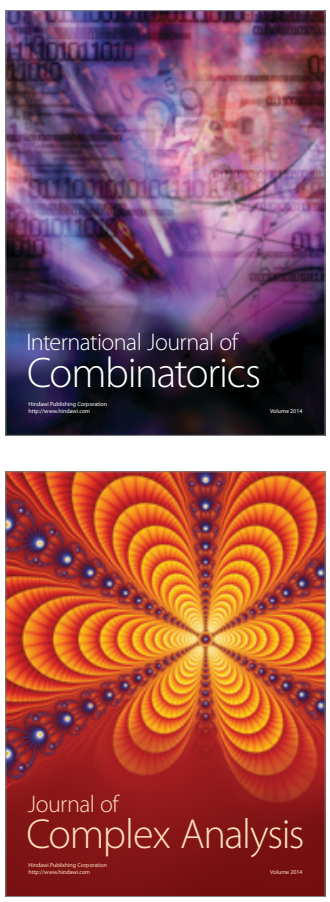

International Journal of

Mathematics and

Mathematical

Sciences
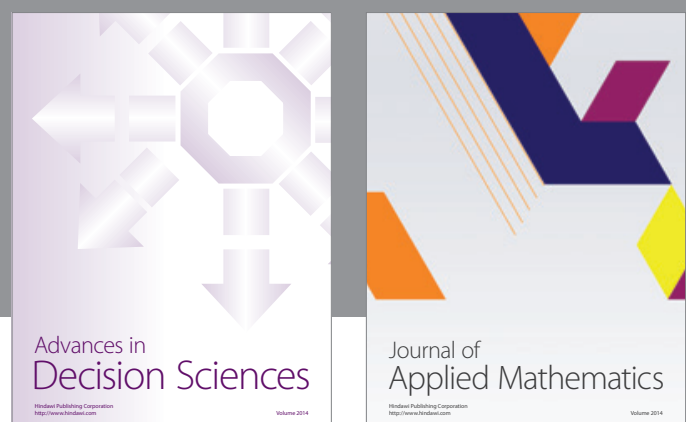

Journal of

Applied Mathematics
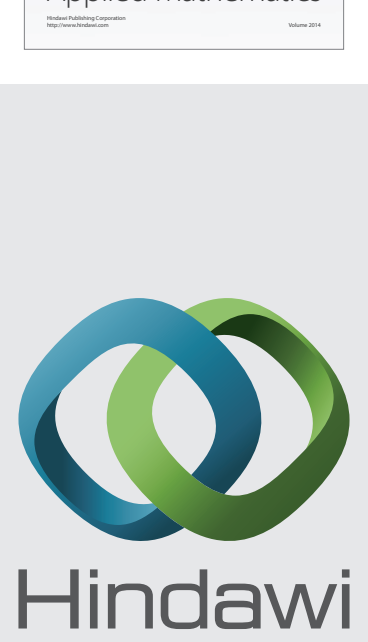

Submit your manuscripts at http://www.hindawi.com
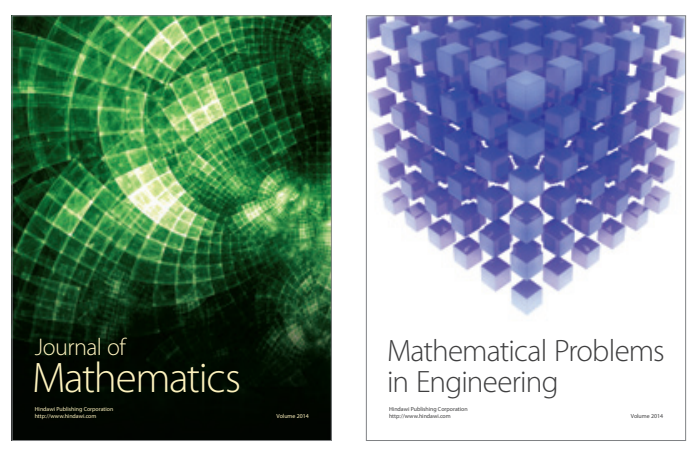

Mathematical Problems in Engineering
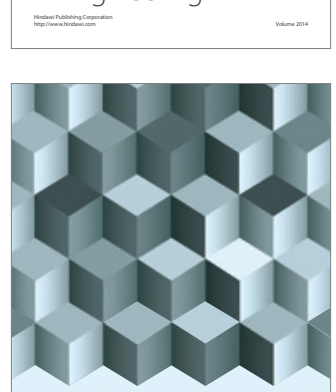

Journal of

Function Spaces
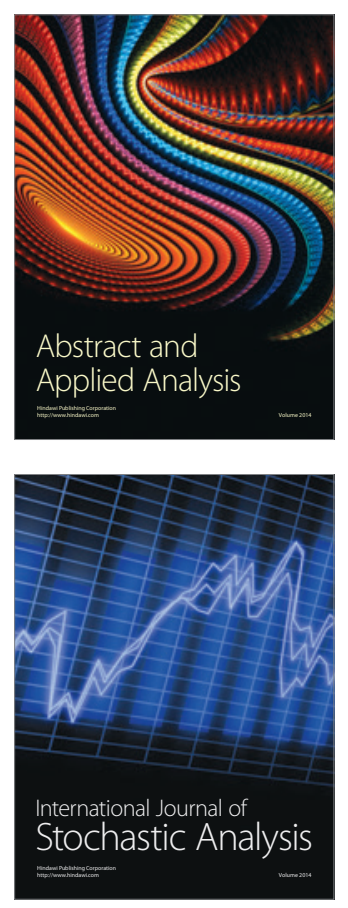

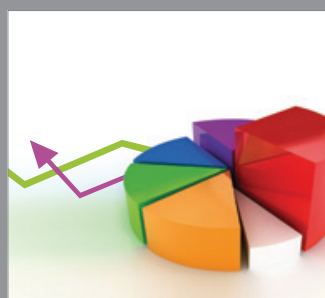

ournal of

Probability and Statistics

Promensencen
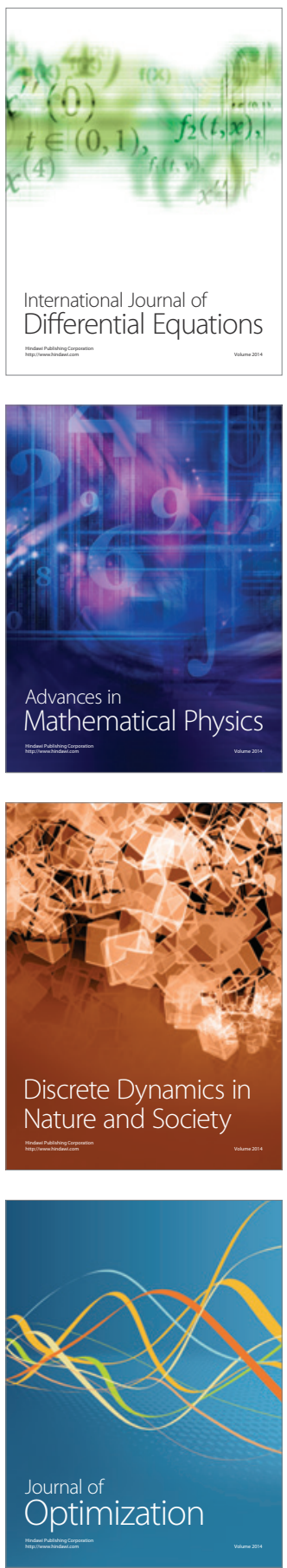